Check for updates

Cite this: RSC Adv., 2017, 7, 27780

Received 31st March 2017

Accepted 15th May 2017

DOI: $10.1039 / c 7 r a 03716 c$

rsc.li/rsc-advances

\section{Structural insight into inhibition of REV7 protein interaction revealed by docking, molecular dynamics and MM/PBSA studies $\uparrow$}

\author{
Xiaodong Ren, (D) ab Rui Zeng, ${ }^{\text {tb }}$ Changwei Wang, ${ }^{c}$ Mingming Zhang, ${ }^{d}$ \\ Chengyuan Liang, ${ }^{e}$ Zhonghai Tang ${ }^{f}$ and Jinfeng Ren ${ }^{g}$
}

\begin{abstract}
In mammalian cells, DNA polymerase $\zeta$ (Pol $\zeta$ ) catalyzes the TLS step of ICLR. By acting simultaneously with Yfamily DNA polymerase, Pol $\zeta$ completes replication of damaged DNA without removing the damage by inserting a nucleotide opposite the lesion. It has been demonstrated that Pol $\zeta$ represents a promising target for the treatment of chemotherapy-resistant tumors. The first series of small-molecule inhibitors targeting REV7/ REV3L interaction have been identified recently, however, their corresponding binding mechanism is not known. Herein, we performed docking, molecular dynamics and MM/PBSA free energy calculations to study the binding mechanism of REV7 and its inhibitors. It was demonstrated that inhibitors bind to the two pockets divided by the 'safety-belt' structure of REV7, which was supported by the MD simulation. In addition, 2-methylfuran is an important group with an appropriate size to form the stable complex, and hydrophobic contacts were mainly responsible for stable complex formation as revealed by free energy calculation.
\end{abstract}

\section{Introduction}

DNA interstrand crosslinks (ICLs) are highly toxic DNA lesions that covalently link two bases on the complementary strands of DNA to prevent transcription and replication by inhibiting DNA strand separation. ${ }^{1}$ Agents that induce ICLs were one of the earliest chemotherapeutic drugs which are still widely used (e.g., Cisplatin). Mammalian cells have extensive mechanisms for ICL repair (ICLR), which include nucleotide excision repair (NER), translesion DNA synthesis (TLS) and homologous recombination (HR). ${ }^{2,3}$

TLS releases the DNA replication blockage by replacing the stalled replicative polymerase with a DNA polymerase specialized for TLS. It is generally considered that TLS includes two steps performed by at least two types of TLS polymerases, namely inserter and extender polymerases., ${ }^{\mathbf{4 5}}$ In the first step,

${ }^{a}$ Department of Pharmacy, Guizhou Provincial People's Hospital, Guiyang 550002, P. R. China

${ }^{b}$ College of Pharmacy, Southwest University for Nationalities, Chengdu 610041, P. R. China.E-mail: rzeng@swun.edu.cn

${ }^{c}$ Guangzhou Institute of Biomedicine and Health (GIBH), Chinese Academy of Sciences (CAS), Guangzhou, Guangdong 510530, P. R. China

${ }^{d}$ School of Pharmacy, Fudan University, Shanghai 201203, P. R. China

${ }^{e}$ Department of Pharmacy, Shaanxi University of Science \& Technology, Xi'an 710021, P. R. China

${ }^{f}$ College of Bioscience and Biotechnology, Hunan Agriculture University, Changsha 410128, Hunan, P. R. China

${ }^{g}$ Department of Medicine, Stony Brook University, Stony Brook, New York. 11794, USA $\uparrow$ Electronic supplementary information (ESI) available. See DOI: $10.1039 / \mathrm{c} 7 \mathrm{ra03716c}$ a Y-family DNA polymerase such as Pol $\eta$, Pol $\kappa$, Pol ı and REV1 functions as inserter polymerase, incorporates a nucleotide opposite the DNA lesion instead of the stalled replicative polymerase at the damage site. ${ }^{6}$ In the second step, Pol $\zeta$ functions as extender polymerase, which extends a few additional nucleotides before a replicative polymerase restarts DNA replication. ${ }^{7}$

Human Pol $\zeta$ is composed of two subunits, REV3L and REV7. REV3L is a large $350 \mathrm{kDa}$ catalytic subunit and is classified as Bfamily DNA polymerase. REV7 is a small $24 \mathrm{kDa}$ non-catalytic subunit of Pol $\zeta$ and interacts with the central region of REV3L, and the interaction is indispensable for the cellular function of REV3L. ${ }^{8}$ Pol $\zeta$ is an important determinant of tumor resistance to chemotherapeutic agents in several types of human cancers. ${ }^{9-13} \mathrm{Pol} \zeta$ expression can be used as the predictor for poor prognosis, which might be caused by the potential chemoradiation resistance of the cervical cancer patients. ${ }^{\mathbf{1 4}}$ Furthermore, downregulation of REV3L expression significantly enhanced the sensitivity of human cancer cells to cisplatin therapy. ${ }^{10,15}$ Therefore, Pol $\zeta$ represents a promising target for the treatment of chemotherapy-resistant tumors.

The large size of human REV3L impedes the development of REV3L inhibitors, as it is difficult to produce REV3L for enzymatic assays, as well as 3D structural elucidation of the fulllength REV3L protein. However, crystal structures of the complex of REV7 with the REV3L(1874-1893) fragment have been determined, ${ }^{\mathbf{8 1 6 - 1 8}}$ which enable the development of small molecules targeting the protein-protein interaction. Structurally, the most notable feature of this interaction is that a portion of the REV3L(1874-1893) is inserted into the bridge-like 'safetybelt' structure of REV7 (Fig. 1). ${ }^{8,17,18}$ 


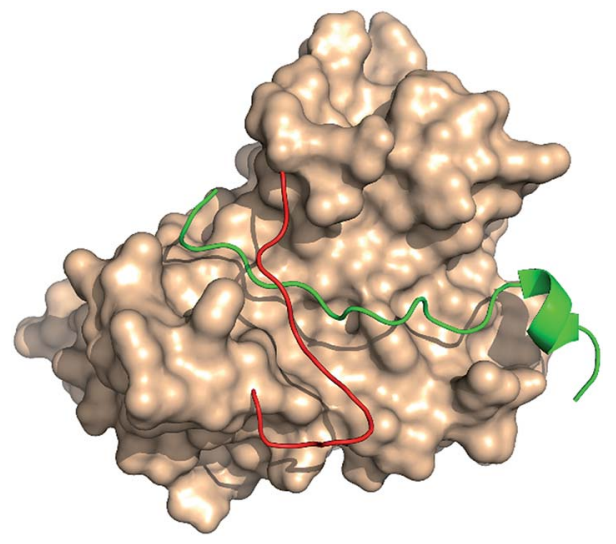

Fig. 1 The complex of REV7 and REV3L fragment. REV7 was showed as surface except for 'safety-belt' (red). REV3L(1874-1893) fragment was showed as green (PDB ID: 4gk0).

In 2016, Fujii et al. conducted a HTS to screen compounds that can inhibit the REV7/REV3L interaction, which resulted in the discovery of compound $\mathbf{1}$ (Fig. 2), an inhibitor of the interaction of REV7 with the REV7-binding sequence of REV3L. ${ }^{19}$ In the thermal shift assay, compound 1 showed a clear shift in the denaturation point of the His-REV7(R124A)/REV3L(1847-1898) complex, suggesting that compound 1 exchanged with REV3L(1847-1898) in the complex but was not nonspecifically bound on the complex. The pull-down assay also indicated that both compound $\mathbf{1}$ and REV3L(1875-1895) can exchange with the biotin-AviTagREV3L(1846-1898) in the complex, resulting in the release of HisREV7(R124A) from the beads. Both assays confirmed that compound 1 is a true inhibitor of the REV7-REV3L(1875-1895) interaction. However, the $\mathrm{IC}_{50}$ of $\mathbf{1}$ is more than $100 \mu \mathrm{M}$. With $\mathbf{1}$ as a lead compound, its analogs 2-17 (Fig. 2) were synthesized and evaluated for REV7/REV3L interaction inhibition. Compound 7, 13, 14 and 15 exhibited a better activity than 1. Compound 7, with an $\mathrm{IC}_{50}$ of $78 \mu \mathrm{M}$, was selected for further investigation. It was demonstrated that 7 bound directly to REV7 in nuclear magnetic resonance analyses, and inhibited the reactivation of a reporter plasmid containing an ICL in between the promoter and reporter regions. Furthermore, the normalized clonogenic survival of HeLa cells treated with cisplatin and compound 7 was lower than that for cells treated with cisplatin only.

The study indicated that a small-molecule inhibitor of the REV7/REV3L interaction can chemosensitize cells by inhibiting ICLR. However, as the first series of REV7 inhibitor, the binding mechanism of the inhibitor to REV7 is not known. Herein, we performed molecular docking, molecular dynamics, and binding free energy calculation to understand the binding mechanism of the inhibitors to the receptor, which will facilitate further design and development of more potent inhibitor targeting REV7/REV3L interaction.

\section{Materials and methods}

\section{Protein and ligand preparation}

The complex of REV7/REV3L was obtained from the RCSB Protein Data Bank (http://www.rcsb.org) with entry code 4 gk0, and only the chain A was kept for further study. ${ }^{16}$ Three missing residues (107-109 aa) which are far from the REV7/REV3L binding interface were built by the 'Model Loops' module of Chimera 1.11.2, ${ }^{20}$ which was subjected to 3 Drefine $^{21}$ for structural refinement and the resulting structure was used for docking study. All of the 3D structure of small molecules were built and energy minimized using Avogadro v1.2.0. ${ }^{22}$

\section{Molecular docking}

AutoDock 4.2.3 program package was used for molecular docking. AutoDock Tools 1.5.6 (ADT) was used to prepare the PDBQT file format of ligands and protein. ${ }^{23}$ The docking calculations were performed by locating a $100 \times 100 \times 66$ points grid map and with a $0.375 \AA$ grid point spacing which covers the entire interface of REV3L/REV7. 250 independent docking runs were performed for each docking simulation with 2500000 energy evaluations for each run. Other docking parameters were set to default. In docking calculations, the<smiles>[R8]c1csc2ncn(CC3CCN(C(C)=O)CC3)c(=O)c12</smiles>

$\mathbf{R}_{1}=$

1

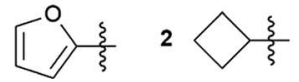

3

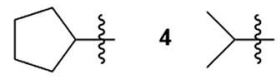

5

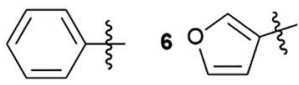<smiles>[R2]c1ccc(-c2csc3ncn(CC4CCN(C(C)=O)CC4)c(=O)c23)o1</smiles>

$\mathbf{R}_{\mathbf{2}}=$
$9\left(\mathrm{CH}_{3}\right)_{2} \mathrm{NHCO}-$

11

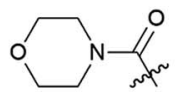

10
$8 \mathrm{CH}_{3} \mathrm{NHCO}$<smiles>CC(C)(C)C(=O)N1CCCC1</smiles>

16<smiles>CC(C)(C)Cc1ccccc1</smiles>

14<smiles>[R]n1cnc2scc(-c3ccc(C)o3)c2c1=O</smiles><smiles>[R]C=[R]</smiles><smiles>CC(=O)N1CCC(CC(C)(C)I)C1</smiles>
15<smiles>CC(=O)N1CCCC(CC(C)(C)C)C1</smiles>
17

Fig. 2 The structure of compound 1 and its analogs 2-17. 
obtained poses were ranked using an energy-based scoring function. After all outputs were clustered based on the root mean squared deviation (RMSD) values, the top pose of docked ligands with the lowest energy in the biggest cluster were saved. For all docking analyses, only the best-scored pose was taken into account.

\section{Molecular dynamics simulation}

MD simulation of the interested complexes were carried out using GROMACS 2016.1 package. ${ }^{24}$ Autodock generated docking conformation of REV7-ligand complexes with lowest binding energy were taken as the initial conformation for MD simulation. The protein was processed by Gromacs with AMBER99SB force field ${ }^{25}$ to generate coordinates and topology files. The ligands were parameterized using a general amber force field $(\mathrm{GAFF})^{26}$ with ACPYPE software, ${ }^{27}$ and AM1-BCC charge model $^{28,29}$ was used to assign charges to the ligands (the GAFF parameters for ligands generated by ACPYPE are provided in the ESI $\dagger$ ). The complex was immersed in a dodecahedron box of TIP3P water molecules using a solute-box distance of $1.0 \mathrm{~nm}$, and periodic boundary condition was used. Energy minimization was performed using the steepest descent method of 10000 steps followed by the conjugate gradient method for 10000 steps to release conflicting contacts. After applying position restraints on protein and inhibitors, NVT equilibration was done at $300 \mathrm{~K}$ and 100 ps of run followed by NPT equilibration of 100 ps with Parrinello-Rahman barostat at reference pressure of 1 bar. After equilibration, production MD run was performed for $20 \mathrm{~ns}$ for each complex. Particle-mesh Ewald algorithm was used for longrange electrostatic interactions, and short-range electrostatics and van der Waals cutoffs were set at $1.4 \mathrm{~nm}$. The atomic coordinates were recorded every 10 ps during the MD simulation. Representative structures were chosen by RMSD-based conformational clustering with the gromos algorithm. All the structural images were generated using Pymol 1.8. ${ }^{30}$

\section{Binding free energy calculation}

The Molecular Mechanics/Poisson-Boltzmann Surface Area (MM/PBSA) method $^{31}$ is the widely used method for binding free energy calculations from the snapshots of MD trajectory. The binding free energies of the complexes between ligands and REV7 were analyzed during equilibrium phase by taking 200 snapshots at an interval of 50 ps from 10 to 20 ns MD simulations using the g_mmpbsa tool. ${ }^{32}$

\section{Results and discussions}

\section{Molecular docking}

In the present study, we applied the molecular docking to gain structural insight into the binding modes of REV7 and its inhibitors. There are 16 analogs (2-17) of compound 1 which were synthesized and evaluated for REV7/REV3L interaction inhibition. The activity results were classified as active, 'modest' and inactive according to the literature (Table 1). For comparison, all of the compounds were docked into the REV7 at the REV3L-binding domain. As there is a chiral center in 14 and 15,
Table 1 The binding energy of compounds binding to REV7 calculated by Autodock

\begin{tabular}{lll}
\hline Compounds & Activity $^{a}$ & Binding energy $\left(\mathrm{kcal} \mathrm{mol}^{-1}\right)$ \\
\hline $\mathbf{1}$ & Modest & -8.98 \\
$\mathbf{2}$ & Inactive & -9.35 \\
$\mathbf{3}$ & Inactive & -9.71 \\
$\mathbf{4}$ & Inactive & -8.73 \\
$\mathbf{5}$ & Inactive & -9.60 \\
$\mathbf{6}$ & Inactive & -8.96 \\
$\mathbf{7}$ & Active & -9.68 \\
$\mathbf{8}$ & Modest & -9.27 \\
$\mathbf{9}$ & Modest & -9.27 \\
$\mathbf{1 0}$ & Inactive & -9.98 \\
$\mathbf{1 1}$ & Modest & -10.57 \\
$\mathbf{1 2}$ & Modest & -6.81 \\
$\mathbf{1 3}$ & Active & -9.72 \\
$\mathbf{1 4 R}$ & Active & -9.42 \\
$\mathbf{1 4 S}$ & & -10.03 \\
$\mathbf{1 5 R}$ & Active & -9.92 \\
$\mathbf{1 5 S}$ & & -9.83 \\
$\mathbf{1 6}$ & Inactive & -9.25 \\
$\mathbf{1 7}$ & Modest & -8.86
\end{tabular}

${ }^{a} \mathrm{IC}_{50}$ values were not provided in the literature except for $7\left(\mathrm{IC}_{50}=78\right.$ $\mu \mathrm{M})$. According to the inhibition results in different concentration, the activity was classified as 'active': compounds inhibit REV7 at $100 \mu \mathrm{M}$; 'modest': compounds inhibit REV7 at $300 \mu \mathrm{M}$; 'inactive': compounds didn't inhibit REV7 at $300 \mu \mathrm{M}$.

both the $R$ and $S$ configurations were investigated in the following studies. The binding energy for each compound was calculated by Autodock and summarized in Table 1. It was demonstrated from the table that the active compounds $(7,13$, $14 R / S, 15 R / S$ ) have an overall lower binding energy. 'Modest' and inactive compounds $1,2,4,6,8,9,12,16$ and 17 have a higher binding energy than all of the active compounds, while only 3, 5, 10 and 11 have a comparable or lower binding energy than the active compounds.

To better analyze the binding pose, we designated the REV7/ REV3L interface as two pockets (Fig. 3A). By positioning the Cterminal to the top and $\mathrm{N}$-terminal to the bottom, with the 'safety-belt' faced with the viewer, the entire groove was divided by the 'safety-belt' (red) as two pockets. The left pocket was named as pocket 1 , while the right one as pocket 2 . As shown in Fig. 3B, the active compounds $13,14 R / S, 15 R / S$ share similar binding pose, while 7 overlapped with them by 2-methylfuran moiety, with its $N$-acetylpiperidine group projected to another direction. Another important feature is that all of the active compounds occupied both the pocket 1 and pocket 2 . Inactive compounds 3, 5 and $\mathbf{1 0}$ or 'modest' compound $\mathbf{1 1}$ have comparable or lower binding energy than the active compounds, but 5, 10, 11 have different binding poses with the active compounds, only compound 3 has a good overlap with 7 (Fig. 3C). The other "modest" or inactive compound 1, 2, 4, 6, 8, 9, 12, 16 and 17 have a higher binding energy, which is consistent with its experimental activity.

By further analyzing the binding mode of compound 1, 3 and 7, we would like to explain the binding process as follow. As shown in Fig. 3D-F, there is a narrow hole (hereafter termed as 

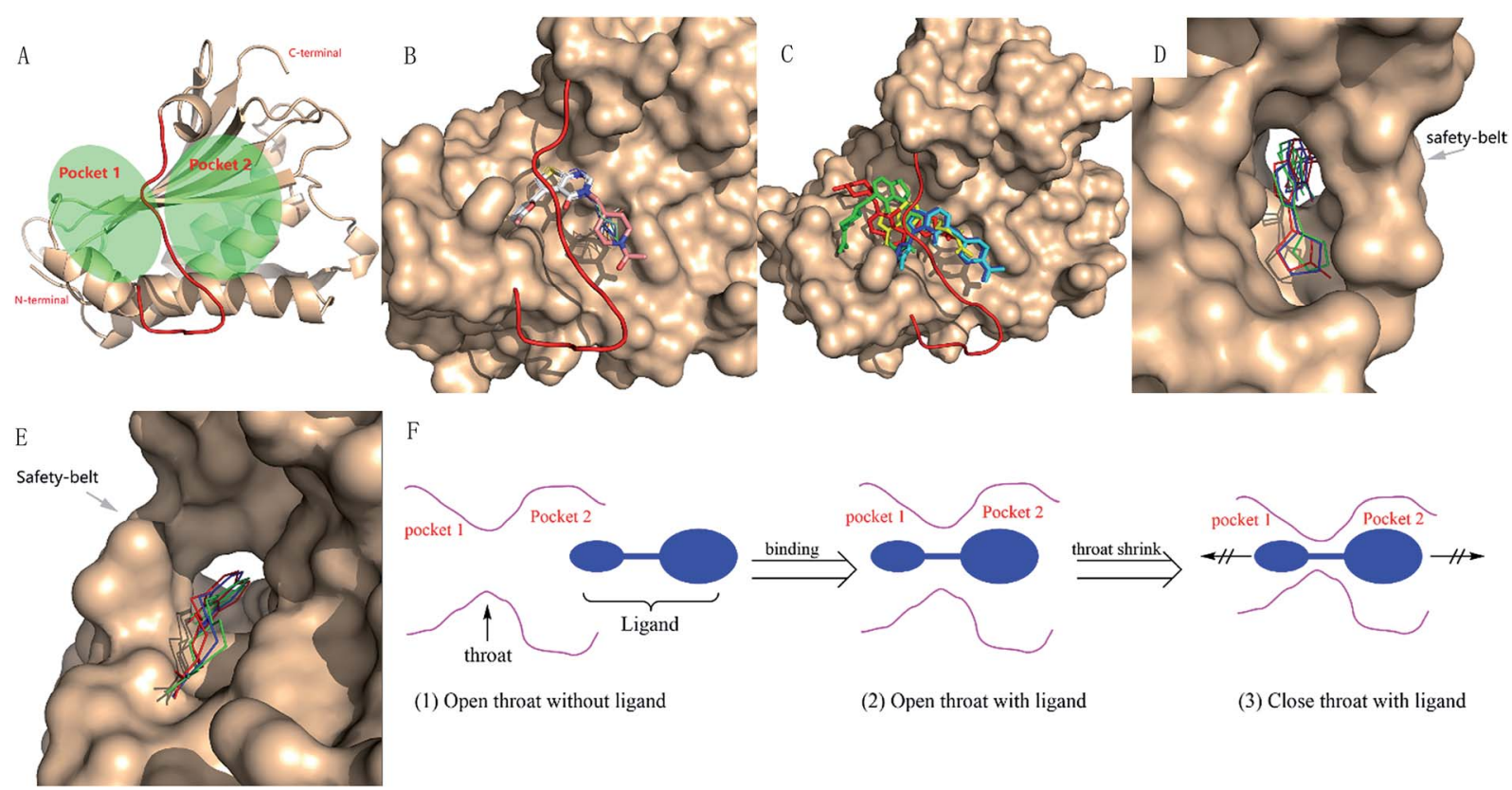

F

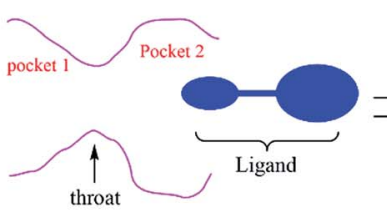

(1) Open throat without ligand

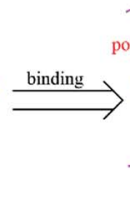

(2) Open throat with ligand

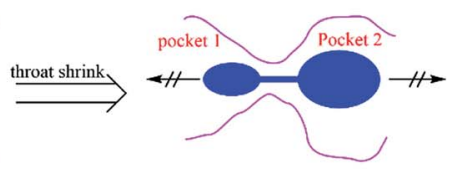

(3) Close throat with ligand

Fig. 3 The REV7 pockets, the binding mode of inhibitors to REV7 and the proposed binding process. (A) Pocket 1 and pocket 2 in REV7 divided by the 'safety-belt' (red). (B) The binding pose of compound 7 (stick) and 13 (line), 14S/R (line), 15S/R (line) in the REV7 pocket. (C) The binding pose of compound 3 (blue, stick), 5 (yellow, stick), 7 (cyan, stick), 10 (red, stick), and 11 (green, stick). (D) Binding mode of compound 1 (green), 3 (blue) and 7 (red) viewed from the pocket 1 side. (E) Binding mode of compound 1 (green), 3 (blue) and 7 (red) viewed from the pocket 2 side. (F) The schematic binding process of inhibitor binding to REV7.

'throat') between pocket 1 and pocket 2. The uncomplexed 'safety-belt' is a highly flexible region which is the major reason that uncomplexed REV7 has not been solved until now. An effective inhibitor must pass through the 'throat' when the 'throat' is in an open state. After passing through the 'throat', pocket 1 and pocket 2 are both occupied by the ligand, then the receptor-ligand forms a stable complex, with the 'throat' shrinking to a close state to avoid the dissociation of ligand from the receptor. All of the active compounds have a 2-methylfuran group, and the methyl group is vital to the activity. Comparison of compounds 1, 3 and 7 indicated that 1, 3 and 7 can pass through the 'throat' from pocket 2 to pocket 1 , compound 7 can form stable complex while $\mathbf{1}$ and $\mathbf{3}$ with less steric hindrance can more easily retreat back from the 'throat' to pocket 2. 'Modest' compound $\mathbf{8}$ and $\mathbf{9}$, with a larger group on furan, are probably less likely to even pass through the 'throat'. When REV3L fragment binds to REV7 and forms a stable complex, it should also undergo such 'insertion-shrink' process. But the REV3L fragment is linear and flexible, its binding to REV7 can be reversible by retreating from the 'throat', as demonstrated by previous results that inhibitor and REV3L(1875-1895) can exchange with the biotin-AviTagREV3L(1846-1898) in the complex.

Although the 3D structure of REV7 complexed with REV3L is relatively a closed state compared with un-complexed REV7. It is reasonable to use the REV3L-binding REV7 in a closed state for docking, as the complex of REV7 with an effective smallmolecule ligand should also undergo a conformation change to form a stable complex. The obtained binding pose and the activity result for each compound can be reasonably explained by the proposed binding process, which will be further investigated by MD simulation.

\section{Molecular dynamics simulation}

Stability of REV7-ligand complexes. To gain insights into the structure and thermodynamics of complex biological systems, 20 ns MD simulation were performed for complexes of REV7 with active compounds $7,13,14 R / S$ and $15 R / S$. To evaluate the reliable stability of the MD trajectories, the root mean square deviation (RMSD) values of the REV7 backbone atoms relative to the initially minimized structure through the phase of the simulation were calculated (Fig. 4). All of the complexes reached

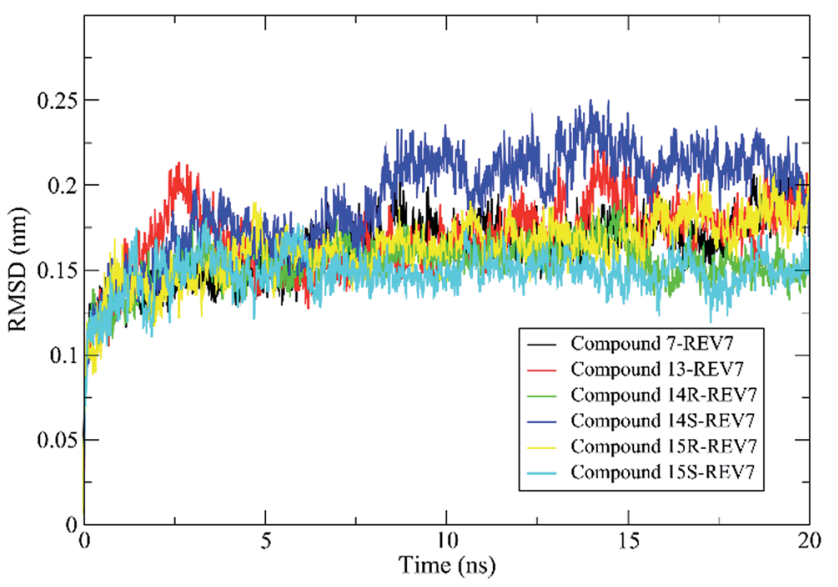

Fig. 4 Root mean square deviations of complexes of REV7 with compounds $7,13,14 R / S, 15 R / S$ along the MD simulation. 
equilibrium after $10 \mathrm{~ns}$ of the simulation phase, which showed that the trajectories of the MD simulations for all of the complexes after equilibrium are reliable for further analyses.

Free energy calculations. The MM/PBSA approach for binding free energy calculation has grown to be one of the most widely used methods to compute interaction energies and is often employed to study biomolecular complexes..$^{33} \mathrm{~g}$ _mmpbsa has been proved to be an effective tool which can be used to estimate the relative binding free energy as well as provide a decomposition of the residue contribution to binding. ${ }^{32}$

In our study, 200 snapshots were extracted at every 50 ps of stable intervals from 10-20 ns MD trajectory. The binding free energy and its corresponding components obtained from the MM/PBSA calculation of the REV7-inhibitor complexes are listed in Table 2. As shown in the table, all of the active compounds with comparative activity have close binding free energies and the difference of binding energies between $R$ and $S$ configuration for $\mathbf{1 4}$ and $\mathbf{1 5}$ are not obvious. It was found that according to free energy calculations, hydrophobic contacts (vdW energy) are a key to REV7 inhibition by this series of inhibitors.

The contribution of residues to the binding energy are also calculated by the g_mmpbsa tool and was shown in Fig. 5. It was demonstrated that residues Leu149, Met160, Ile163, Phe169, Trp171, Ile172, and Leu173 are the key residues for most of the active compounds binding to REV7. It has been demonstrated that Trp171 in REV7 are crucial for the physical interaction with
REV3L by previous in vitro binding assays using alanine mutants of $\operatorname{REV} 7,{ }^{8}$ which is consistent with our result that Trp171 contributes the most binding energy for all of the active compounds.

Interactions between inhibitors and REV7. The interactions between inhibitors and REV7 of each simulated system were investigated in detail with the equilibrium trajectories of MD simulations. 500 snapshots extracted from the last $10 \mathrm{~ns}$ equilibrium trajectory with an interval of 20 ps were clustered, and the center structure of the biggest cluster was selected as the representative conformation of each simulated system. All the 6 representative complexes are compared with its initial structure to analyze the conformational change of the complexes (Fig. 6). As shown in the figures, 'safety-belt' in all complexes exhibited a big fluctuation. In 7 and $15 R$ complexed REV7, the 'safety-belt' moved toward the pocket 1 , while in $13,14 R, 14 S$ and $15 S$ complexed REV7, it moved toward the pocket 2 . In all of the cases, the ligands occupied both pocket 1 and pocket 2 , without skidding out to one pocket through the 'throat'. 2D schematic diagrams of protein-ligand interactions for the representative MD simulated complexes are shown in Fig. 6B, D, F, H, J and L. At least one hydrogen bond was formed for all of the complexes, and in the complex of REV7 with $\mathbf{1 3}$, four hydrogen bonds were formed. However, hydrophobic contacts still play the major role in the protein-ligand interaction.

Molecular dynamics simulations of pharmaceutically relevant protein targets in complex with (putative) ligands have

Table 2 Average MM/PBSA free energies of REV7-inhibitor complexes calculated from the MD simulations

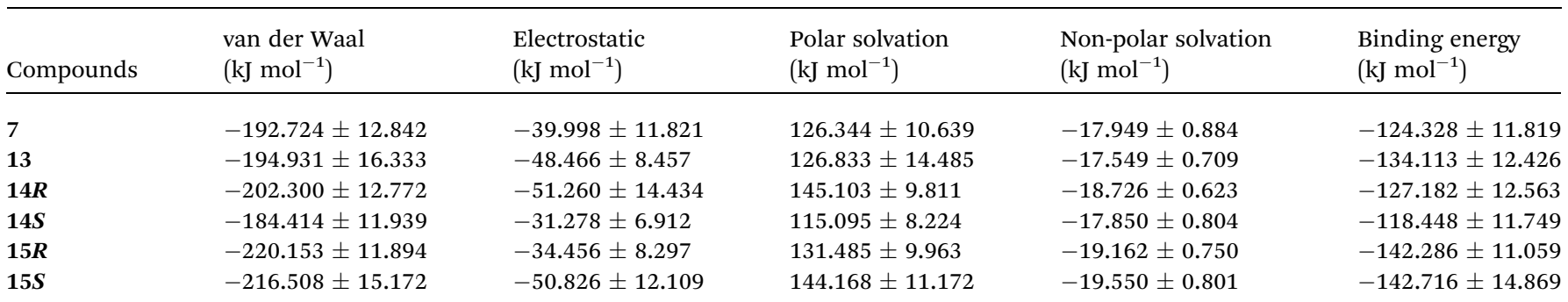

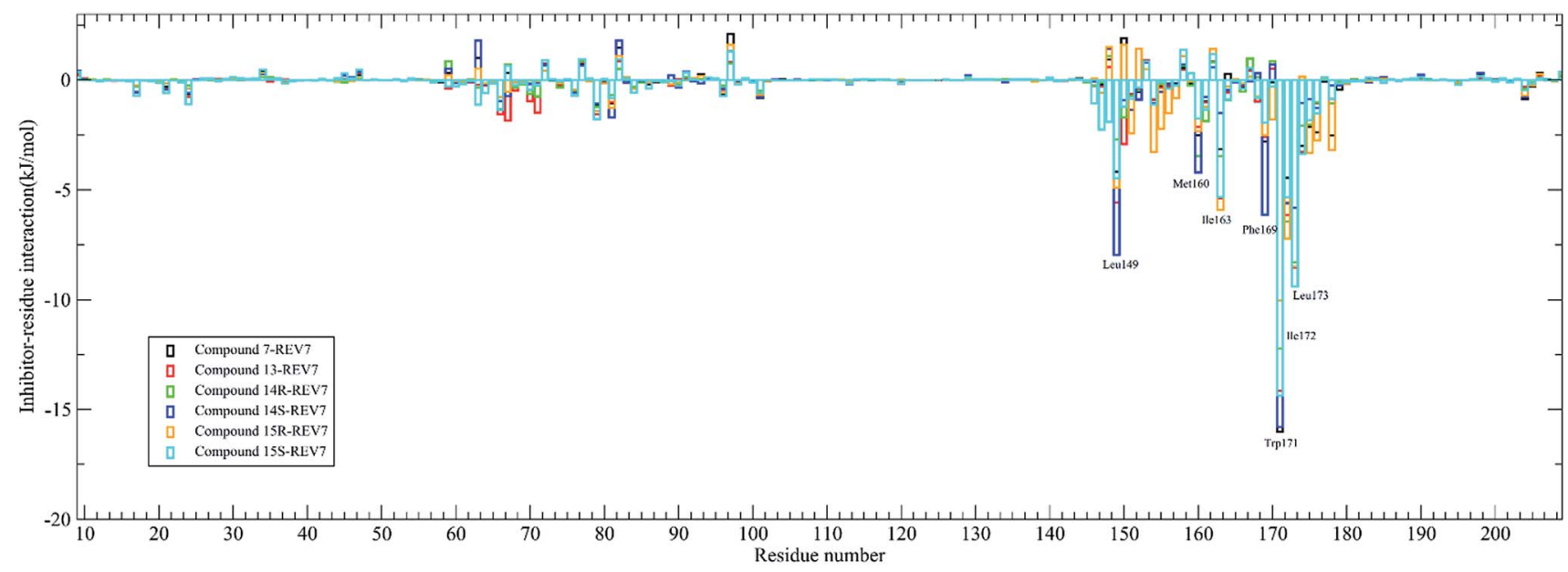

Fig. 5 The contribution of residues to the binding energy for active compounds. 

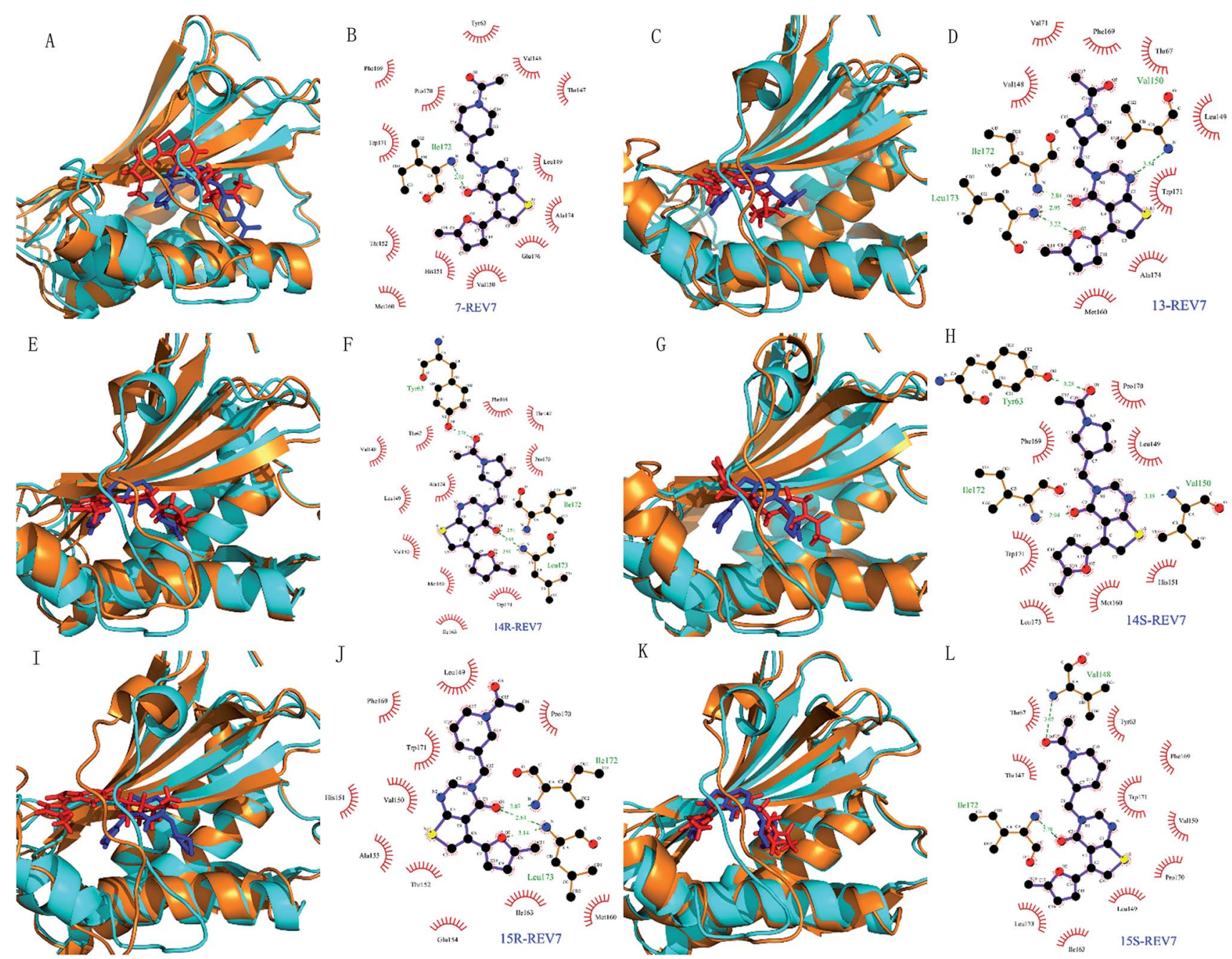

Fig. 6 Conformation change of the simulated complexes and interaction mode for representative complex. (A, C, E, G, I and K) are complexes of REV7 with compounds $7,13,14 R, 14 S, 15 R$, and 15S. For each comparison diagram, representative MD simulated REV7 (orange) and ligands (red) were aligned to the initial REV7 structure (cyan) and ligands (blue). (B, D, F, H, J and L) are 2D schematic diagrams for MD simulated representative complexes of REV7 with 7, 13,14R,14S, 15R and $15 S$ generated by Ligplot v.4.5.3. ${ }^{34}$ In the 2D schematic diagrams, hydrogen bonds are indicated by dashed lines between the atoms involved, while hydrophobic contacts are represented by an arc with spokes radiating towards the ligand atoms they contact. The contacted atoms are shown with spokes radiating back.

provided useful information in drug design, and several examples using MD simulations for the drug design have been reviewed. ${ }^{35}$ In this study, molecular dynamics was used for validation of predicted binding mode. All of the complexes of REV7 and active compounds reached equilibrium after $10 \mathrm{~ns}$ of the simulation phase revealed by RMSD, which showed that the trajectories of the MD simulations for all of the complexes after equilibrium are reliable for further analyses. All of the representative complexes showed that although the 'safety-belt' have a big fluctuation, the 2-methylfuran moiety stay in the pocket 1 , without skidding out to pocket 2 through the 'throat', which support our hypothesis of binding mode shown in Fig. 3F.

\section{Conclusion}

In the present study, the binding pattern of REV7/REV3L protein interaction and its first series of inhibitors were investigated by using molecular docking, molecular dynamics as well as MM/PBSA free energy calculation. Molecular docking revealed that all active compounds $(7,13,14 R / S, 15 R / S)$ bind to the two pockets divided by 'safety-belt' structure of REV7 and the bindings were found to be stable throughout MD simulation. The hydrophobic contacts were mainly responsible for stable complex formation as revealed by the vdW energy and Ligplot analysis. 2-Methylfuran is an appropriate group to both passing through the 'throat' and form a stable complex with REV7 in the 'safety-belt' region.

The recently identified inhibitors for REV7/REV3L protein interaction provided hope for developing small-molecule inhibitors targeting Pol $\zeta$ for the treatment of chemotherapyresistant tumors. Limited activity data, as well as lacking of 3D structure of uncomplexed REV7 make it difficult to investigate the binding mechanism. However, the present study still provides important structural insight into the binding 
mechanism of REV7 and its inhibitors which will facilitate further anticancer drug design and development that targeting inhibition of REV7 protein interaction.

\section{Acknowledgements}

This work was funded by the Program of Study Abroad for Young Scholars sponsored by China Scholarship Council (CSC201500850007), Sichuan Province Department of Basic Research Project (2015jy0009), Key Technologies R \& D Program of Sichuan (2014SZ0131), the National Natural Science Foundation of China (Grant No. 81602967) and the China Postdoctoral Science Foundation (Grant No. 2016M592898XB).

\section{References}

1 A. J. Deans and S. C. West, Nat. Rev. Cancer, 2011, 11, 467480.

2 C. Clauson, O. D. Scharer and L. Niedernhofer, Cold Spring Harbor Perspect. Biol., 2013, 5, a012732.

3 A. J. Deans and S. C. West, Nat. Rev. Cancer, 2011, 11, 467480.

4 G.-L. Moldovan, B. Pfander and S. Jentsch, Cell, 2007, 129, 665-679.

5 E. C. Friedberg, A. R. Lehmann and R. P. P. Fuchs, Mol. Cell, 2005, 18, 499-505.

6 H. Ohmori, E. C. Friedberg, R. P. P. Fuchs, M. F. Goodman, F. Hanaoka, D. Hinkle, T. A. Kunkel, C. W. Lawrence, Z. Livneh, T. Nohmi, L. Prakash, S. Prakash, T. Todo, G. C. Walker, Z. Wang and R. Woodgate, Mol. Cell, 2001, 8, 7-8.

7 A. V. Makarova and P. M. Burgers, DNA Repair, 2015, 29, 4755.

8 K. Hara, H. Hashimoto, Y. Murakumo, S. Kobayashi, T. Kogame, S. Unzai, S. Akashi, S. Takeda, T. Shimizu and M. Sato, J. Biol. Chem., 2010, 285, 12299-12307.

9 S. Sharma, N. A. Shah, A. M. Joiner, K. H. Roberts and C. E. Canman, Mol. Pharmacol., 2012, 81, 778-787.

10 H. Wang, S.-Y. Zhang, S. Wang, J. Lu, W. Wu, L. Weng, D. Chen, Y. Zhang, Z. Lu, J. Yang, Y. Chen, X. Zhang, X. Chen, C. Xi, D. Lu and S. Zhao, Neuro-Oncology, 2009, 11, 790-802.

11 W. P. Roos, A. Tsaalbi-Shtylik, R. Tsaryk, F. Güvercin, N. de Wind and B. Kaina, Mol. Pharmacol., 2009, 76, 927.

12 X. Lin, J. Trang, T. Okuda and S. B. Howell, Clin. Cancer Res., 2006, 12, 563.

13 F. Wu, X. Lin, T. Okuda and S. B. Howell, Cancer Res., 2004, 64, 8029.

14 T.-Y. Shi, L. Yang, G. Yang, X.-Y. Tu, X. Wu, X. Cheng and Q. Wei, Med. Oncol., 2013, 30, 500.
15 M. Adachi, K. Ijichi, Y. Hasegawa, T. Ogawa, H. Nakamura, Y. Yasui, M. Fukushima and K. Ishizaki, Mol. Med. Rep., 2008, 1, 695-698.

16 W. Xie, X. Yang, M. Xu and T. Jiang, Protein Cell, 2012, 3, 864-874.

17 J. Wojtaszek, C.-J. Lee, S. D'Souza, B. Minesinger, H. Kim, A. D. D'Andrea, G. C. Walker and P. Zhou, J. Biol. Chem., 2012, 287, 33836-33846.

18 S. Kikuchi, K. Hara, T. Shimizu, M. Sato and H. Hashimoto, J. Biol. Chem., 2012, 287, 33847-33852.

19 M. L. Actis, N. D. Ambaye, B. J. Evison, Y. Shao, M. Vanarotti, A. Inoue, E. T. McDonald, S. Kikuchi, R. Heath, K. Hara, H. Hashimoto and N. Fujii, Bioorg. Med. Chem., 2016, 24, 4339-4346.

20 E. F. Pettersen, T. D. Goddard, C. C. Huang, G. S. Couch, D. M. Greenblatt, E. C. Meng and T. E. Ferrin, J. Comput. Chem., 2004, 25, 1605-1612.

21 D. Bhattacharya, J. Nowotny, R. Cao and J. Cheng, Nucleic Acids Res., 2016, 44, W406-W409.

22 M. D. Hanwell, D. E. Curtis, D. C. Lonie, T. Vandermeersch, E. Zurek and G. R. Hutchison, J. Cheminf., 2012, 4, 17.

23 G. M. Morris, R. Huey, W. Lindstrom, M. F. Sanner, R. K. Belew, D. S. Goodsell and A. J. Olson, J. Comput. Chem., 2009, 30, 2785-2791.

24 M. J. Abraham, T. Murtola, R. Schulz, S. Páll, J. C. Smith, B. Hess and E. Lindahl, SoftwareX, 2015, 1-2, 19-25.

25 V. Hornak, R. Abel, A. Okur, B. Strockbine, A. Roitberg and C. Simmerling, Proteins: Struct., Funct., Bioinf., 2006, 65, $712-725$.

26 J. Wang, R. M. Wolf, J. W. Caldwell, P. A. Kollman and D. A. Case, J. Comput. Chem., 2004, 25, 1157-1174.

27 A. W. Sousa da Silva and W. F. Vranken, BMC Res. Notes, 2012, 5, 367.

28 A. Jakalian, D. B. Jack and C. I. Bayly, J. Comput. Chem., 2002, 23, 1623-1641.

29 A. Jakalian, B. L. Bush, D. B. Jack and C. I. Bayly, J. Comput. Chem., 2000, 21, 132-146.

30 The PyMOL Molecular Graphics System, Version 1.8, Schrödinger, LLC.

31 P. A. Kollman, I. Massova, C. Reyes, B. Kuhn, S. Huo, L. Chong, M. Lee, T. Lee, Y. Duan, W. Wang, O. Donini, P. Cieplak, J. Srinivasan, D. A. Case and T. E. Cheatham, Acc. Chem. Res., 2000, 33, 889-897.

32 R. Kumari, R. Kumar and A. Lynn, J. Chem. Inf. Model., 2014, 54, 1951-1962.

33 N. Homeyer and H. Gohlke, Mol. Inf., 2012, 31, 114-122.

34 A. C. Wallace, R. A. Laskowski and J. M. Thornton, Protein Eng., Des. Sel., 1995, 8, 127-134.

35 H. Zhao and A. Caflisch, Eur. J. Med. Chem., 2015, 91, 4-14. 